\title{
Cross-Linked Polyelectrolyte for Improved Selectivity and Processability of lontronic Systems
}

Theresia Arbring Sjöström, Amanda J onsson, Erik Gabrielsson, Loig Kergoat, Klas Tybrandt, Magnus Berggren and Daniel Simon

The self-archived postprint version of this journal article is available at Linköping University Institutional Repository (DiVA):

http:// urn.kb.se/ resolve?urn=urn:nbn:se:liu:diva-142182

N.B.: When citing this work, cite the original publication.

Arbring Sjöström, T., J onsson, A., Gabrielsson, E., Kergoat, L., Tybrandt, K., Berggren, M., Simon, D., (2017), Cross-Linked Polyelectrolyte for Improved Selectivity and Processability of lontronic Systems, ACS Applied Materials and Interfaces, 9(36), 30247-30252. https:/ / doi.org/ 10.1021/ acsami.7b05949

Original publication available at:

https:// doi.org/ 10.1021/acsami.7b05949

Copyright: American Chemical Society

http:// pubs.acs.org/ 


\section{A crosslinked polyelectrolyte for improved selectivity and processability of iontronic systems}

Theresia Arbring Sjöström, Amanda Jonsson ${ }^{\dagger}$, Erik Gabrielsson, Loïg Kergoat ${ }^{\ddagger}$, Klas Tybrandt, Magnus Berggren and Daniel T. Simon*

Laboratory of Organic Electronics, Department of Science and Technology, Linköping University, 60174 Norrköping, Sweden.

KEYWORDS: Ion exchange membranes, iontronics, organic bioelectronics, microfabrication, neurotransmitter release 


\section{ABSTRACT}

On-demand local release of biomolecules enables fine-tuned stimulation for the next generation of neuromodulation therapies. Such chemical stimulation is achievable using iontronic devices based on microfabricated, highly-selective ion exchange membranes (IEMs). Current limitations in processability and performance of thin film IEMs hamper future developments of this technology. Here we address this limitation by developing a cationic IEM with excellent processability and ionic selectivity: poly(4-styrenesulfonic acid-co-maleic acid) (PSS-co-MA) crosslinked with polyethylene glycol (PEG). This enables new design opportunities and provides enhanced compatibility with in vitro cell studies. PSSA-co-MA/PEG is shown to out-perform the cation selectivity of the previously used iontronic material.

Devices that interact directly with the nervous system represent an emerging medicaltechnology platform enabling therapies for disorders related to dysfunctional neural signaling, including epilepsy ${ }^{1}$, Parkinson's disease ${ }^{2}$, neuropathic pain ${ }^{3}$ and spinal cord injury ${ }^{4}$. Typically, such devices use electrical stimulation from implanted electrodes to restore damaged neural circuits, or to suppress disease-related signaling. An alternative approach to these techniques is to release specific bio-signaling molecules, locally and on-demand, targeting only the subset of cells expressing the receptor for the specific neurotransmitter. Thereby, a relatively higher therapeutic efficacy is achieved as compared to electrical stimulation, since side effects due to the spread and non-specificity of electrical stimulation may be avoided. Iontronic devices can be used for such local, on-demand chemical delivery.,6 Iontronics are a class of technologies and circuit components where significant parts of circuits process and transport ions, rather than 
electrons, as the charged signal carrier. ${ }^{7}$ This performance is based on electrophoretic transport of charged biomolecules through microfabricated polyelectrolyte channels with ion exchange membrane (IEM) properties. These channels allow selective electrophoresis of cations or anions, depending on the sign of the fixed charges of the polyelectrolyte; a cation exchange membrane (CEM) includes fixed negative charges, and selectively transports cations, and vice versa for an anion exchange membrane (AEM).

Iontronic devices can control ion fluxes, for example; the organic electronic ion pump (OEIP) transduces electronic control signals into local release of precise doses of chemical compounds. ${ }^{5}$ The OEIP has shown great potential in a variety of applications, including cell-resolution drug delivery ${ }^{8}$, nerve-injury induced pain $^{6}$, suppression of epileptiform activity ${ }^{9}$ and even regulation of plant physiology via hormone delivery ${ }^{10}$. Iontronics has also expanded to include more complex and non-linear devices, such as ion bipolar membrane diodes (IBMD) $)^{11,12}$ and ion bipolar junction transistors (IBJT) ${ }^{13,14}$. These devices can be used to build chemical delivery circuits, exemplified by an ion diode bridge ${ }^{15}$, $\operatorname{logics}$ gates ${ }^{16}$, and addressable chemical delivery arrays $^{17}$.

Initially, the CEM channel of the OEIPs was based on the commercially available conducting polymer poly(3,4-ethylenedioxythiophene) (PEDOT) blended with the charge-compensating polyanion polystyrene sulfonate (PSS) and pre-coated on polyethylene terephthalate (PET) sheets (Agfa Orgacon ${ }^{\mathrm{TM}}$ ). In those PEDOT:PSS-based devices, the PSS phase provide CEM and the resulting devices have successfully been used to transport various alkali ion and charged neurotransmitters. As an alternative to PEDOT:PSS, poly(4-styrenesulfonic acid-co-maleic acid) PSS-co-MA, a CEM widely used in proton exchange membranes for fuel cell applications ${ }^{18}$, has recently been included in wound-healing applications ${ }^{19}$ and iontronic devices ${ }^{9,17}$. Maleic-acid- 
containing co-polymers can be crosslinked by polymers displaying alcohols such as polyethylene glycol (PEG), by the formation of ester bonds ${ }^{20}$ (Figure 1a). Like the complementary AEMs found in IBMDs and IBJTs ${ }^{12,14}$, PSS-co-MA/PEG is processed from solution by spin coating, which makes fabrication more flexible.

Here, we report a detailed study of PSS-co-MA/PEG by investigating its suitability as the active material in microfabricated iontronic devices. This study includes fabrication of OEIPs on glass and plastic substrates using standard thin film processing protocols. We demonstrate the resulting devices' improved optical characteristics for use in in vitro microscopy cell studies. Fundamental ion transport characteristics such as cation selectivity are investigated and compared with previously used PEDOT:PSS-based CEMs.

First, to evaluate the photolithography compatibility of PSS-co-MA/PEG-based devices, square-shaped test patterns and a multi-outlet OEIP were fabricated on glass. The substrate was first treated with an adhesion promoter 3-glycidoxypropyltrimethoxysilane (GOPS). After deposition and cross-linking, the material does not delaminate, dissolve, swell extensively, or crack during immersion in developer, deionized water, and organic solvents such as acetone and isopropanol.

To visualize the utility of PSS-co-MA/PEG for in vitro experiments on cells (i.e., microscopy), human umbilical vein endothelial cells were seeded and cultured on autoclaved and poly-llysine-treated OEIP devices (Figure 2). The same cell culture procedure was also performed on dry-etched Orgacon $^{\mathrm{TM}}$ foil (PET substrate) for comparison. We find that PSSA-co-MA/PEG on glass can offer a significantly better platform of in vitro/microscopy experiments as compared to Orgacon $^{\mathrm{TM}}$ due to the comparatively fewer visible defects on the glass substrate than the flexible plastic substrates. Furthermore, PSSA-co-MA has been considered non-toxic while crosslinked 
with polyvinyl alcohol (PVA) ${ }^{19}$ and PEG is widely used as a biocompatible polymer ${ }^{21}$. Although this is promising, the biocompability of PSSA-co-MA/PEG should be further investigated prior to future in vitro or in vivo studies.

The selectivity of the IEM for either cations or anions is of utmost importance for proper iontronic device function, and is dictated by the concentration of fixed charges in the IEM relative to the concentration of the adjacent electrolytes..$^{22}$ Selectivity can be quantified using the transport number of the ion intended for transport, defined by the fraction of the current carried by that ion ( $t_{+}$for the cationic transport number and $t$ - for the anionic transport number). The transport number can thus take values between zero and one ( $t_{+}=1$ for an ideal CEM).

To evaluate the transport number of PSS-co-MA/PEG, simple OEIPs including two electrolytes ("source” and "target”) separated by CEM channel(s) were fabricated (Figure 1b). The source electrolyte functions as a reservoir containing the ions intended for transport, whereas the target electrolyte contains the system to which ions are to be delivered. To induce electrophoretic transport of cations, positive electrical potential was applied to the source side, relative to the target side, using $\mathrm{Ag} / \mathrm{AgCl}$ electrodes. The ion channel was encapsulated by the hydrophobic photoresist SU-8, which also served as a barrier and gasket compartments, defining the size of the source and target electrolytes. A low-resistance version (Figure 1c) was used to measure membrane potential to reduce equilibration time and noise, while another version (Figure 1d) closer to general iontronic device design, was used for all other characterizations. Detailed description of all transport characterizations methods can be found in the Supporting Information.

The total ionic current through an IEM is the sum of the cationic and the anionic currents ${ }^{23-25}$. For a perfectly cation-permselective CEM, the concentration of mobile anions within the 
membrane is zero, and all current is carried by cations. Thus, the conductivity should be dependent on the diffusion coefficient of the cation (in the source) and the properties of the anion in the target should ideally not affect the conductivity. ${ }^{26} \mathrm{~A}$ first estimation of the selectivity of a IEM can thus be obtained by observing the change in conductivity for different cation/anion pairs in the source/target electrolyte of an OEIP, and compare how they scale with diffusion coefficients in infinite solution ${ }^{27}$. For PSS-Co-MA/PEG, the resulting conductivites increased with increased diffusion coefficients in the order $\mathrm{K}^{+}>\mathrm{Na}^{+}>\mathrm{Li}^{+}$for source ions (Figure 3a), but showed less dependence on the diffusion coefficients of the target anion where $\mathrm{Cl}^{-}>\mathrm{ClO}_{4}^{-}>\mathrm{OAc}^{-}$ (Figure 3b), suggesting cations are preferentially transported through the CEM ion channel. Similar results were obtained for OEIPs based on the previously used CEM ion channel material, i.e. over-oxidized Orgacon $^{\mathrm{TM}}$ (Figure 3a and b). It should be noted that this method does not account for changes in diffusion coefficent due to, e.g., morphology of the membrane or counterion binding affinity ${ }^{23,28,29}$. Also, the resistance of the ion channel in OEIPs tends to drift during protracted measurements. To investigate whether OEIP devices based on PSS-co-MA/PEG could also transport larger biologically relevant substances, $\mathrm{Ca}^{2+}$, acetylcholine $\left(\mathrm{ACh}^{+}\right)$, carbachol $\left(\mathrm{C}^{+}\right)$, and gamma-aminobutyric acid (GABA) were tested. The biomolecules were all successfully transported with the following conductivities (at the following concentrations): $\mathrm{Ca}^{2+} 6.4 \mathrm{mS} \mathrm{cm}^{-1}$ (0.1 M); GABA: $4.5 \mathrm{mS} \mathrm{cm}^{-1}(0.1 \mathrm{M})$; $\mathrm{ACh}^{+} 5.3 \mathrm{mS} \mathrm{cm}{ }^{-1}(0.1 \mathrm{M})$; and $\mathrm{C}^{+}$; $3.9 \mathrm{mS} \mathrm{cm}^{-1}(0.01$ M).

Next, we estimated the transport number by measuring the membrane potential, also known as the EMF-method ${ }^{30-32}$ (electromotive force). Here, electrolytes of different concentrations were placed on each side of the IEM and the membrane potential was measured. Higher concentration of fixed charges in the IEM, relative to the electrolyte concentration, will result in a more 
pronounced effect of counter-ion inclusion and co-ion exclusion, which is reflected in the magnitude of the Donnan potential. By using $\mathrm{KCl}$ electrolytes and $\mathrm{Ag} / \mathrm{AgCl} / \mathrm{KCl}$ (saturated in water) electrodes, the over-all membrane potential is practically reduced to the two Donnan potentials at the IEM-electrolyte interfaces and the membrane potential $(E)$ is related to the

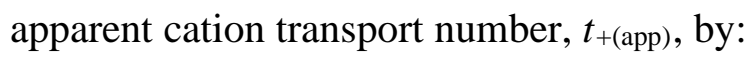

$$
E=\left(1-2 t_{+(a p p)}\right) \frac{R T}{F} \ln \left[\frac{c_{\text {high }}}{c_{\text {low }}}\right]
$$

where $R$ is the universal gas constant $\left(8.3144 \mathrm{~J} \mathrm{~mol}^{-1} \mathrm{~K}^{-1}\right), T$ is the temperature in Kelvin, $F$ Faraday's constant $\left(96485 \mathrm{C} \mathrm{mol}^{-1}\right)$, and $c_{\text {high }}$ and $c_{\text {low }}$ the electrolyte concentrations on the high and low concentration side. ${ }^{30}$ The transport number obtained by this method is commonly referred to as the apparent transport number $\left(t_{+(\text {app })}\right.$ and $\left.t_{\text {-(app) })}\right)$. This method lacks correction for water transport, that occurs in the direction of the major charge carriers and thereby increases their flux, which typically underestimates the actual transport number. ${ }^{30} \mathrm{KCl}$ electrolytes with a concentration difference of a factor of two were used. Measured $E$ and calculated $t_{+(\text {app })}$ for PSSco-MA/PEG-based and Orgacon $^{\mathrm{TM}}$-based OEIPs were plotted as a function of the mean concentration of the two electrolytes (Figure 3c). The two materials perform similarly at low electrolyte concentration $(<0.2 \mathrm{M})$, including physiological salt concentrations. However, it appears that PSS-co-MA/PEG exhibits relatively higher selectivity for cations at increased concentrations. This is of importance, since the concentration in the vicinity of the channel increases as current passes through the channel, a phenomenon known as concentration polarization, which can lead to altered ion transport numbers. ${ }^{22}$ For concentrations below $0.1 \mathrm{M}$, the membrane potential measurements were unstable and sensitive to noise.

Finally, we measured the transport number of the neurotransmitter acetylcholine $\left(\mathrm{ACh}^{+}\right)$ in PSS-co-MA/PEG using an adapted version of the Hittorf method ${ }^{24}$. This method involves the 
direct measurement of changes in ion concentrations due to the passage of ionic currents. We have previously used this method to analyze the transport number of e.g. Glu, Asp and GABA via quantitative HPLC analysis ${ }^{5}$, GABA with mass spectrometry ${ }^{6}$ and, as here, $\mathrm{ACh}^{+}$using an enzymatic-reaction $\mathrm{kit}^{8}$. In brief, $\mathrm{AChCl}$ was used as the source electrolyte, and different amounts of charge were run through the circuit. The target electrolyte was then collected, and the resulting $\mathrm{ACh}^{+}$concentration was measured. By comparing the expected number of transported $\mathrm{ACh}^{+}$(from the measured electronic charge) with the measured amount, the transport number was estimated. Using this method, we estimated the transport number of $\mathrm{ACh}^{+}$to be 0.84 with a $\pm 6 \%$ s.d. (Figure 3d).

In conclusion, the stability during processing and sterilization, the ability to utilize standard thin-film processing protocols on glass, successful cell culturing and imaging, good conductivity for several biomolecules and high a cation selectivity over desired concentration in combination makes PSS-co-MA/PEG a suitable candidate to for future iontronic applications. Reliable techniques for evaluating selectivity of new iontronic materials are essential for the development of new iontronic and bioelectronics delivery devices. By using the three complementary techniques suggested above, a strong statement of a material's selectivity can be established. Traditionally, these methods are performed using vertical architectures with freestanding membranes. Lateral evaluation, as executed here, is preferred for applications and/or materials that need support substrates and where ion transport occurs laterally. PSS-coMA/PEG's solution processability provides several degrees of freedom regarding the design of new, sophisticated iontronic devices. ${ }^{17}$ The material can be patterned on a wide range of substrates and can also be included in between any layer of the targeted device architectures. In some cases, an adhesion promoter may be required for good processability and to avoid leakage 
currents $^{33}$ that reduce or prevent ion selectivity. In addition, covalent bonding between the CEM and the substrate and/or encapsulation material ${ }^{34}$ can also be considered for future devices. We also foresee that techniques such as dip coating or printing can be used to manufacture for instance pipette-like versions of the OEIP ${ }^{6}$. Furthermore, the thickness and the degree of crosslinking can also be tailored for a specific compound. Varying the thickness allows tuning of the resistance, resulting in increased geometric freedom when designing iontronic resistor networks. Varying the amount of cross-linking could be used to tune the swelling of the material. Also, the composition of the material (PSS:MA:PEG ratio) could allow for a precise tuning of the selectivity. This is valuable, for example, in developing bipolar membrane diodes for delivery of biomolecules $^{17}$, as it allows the construction of selective diodes, leading to better control of the delivered amount. 


\section{FIGURES}

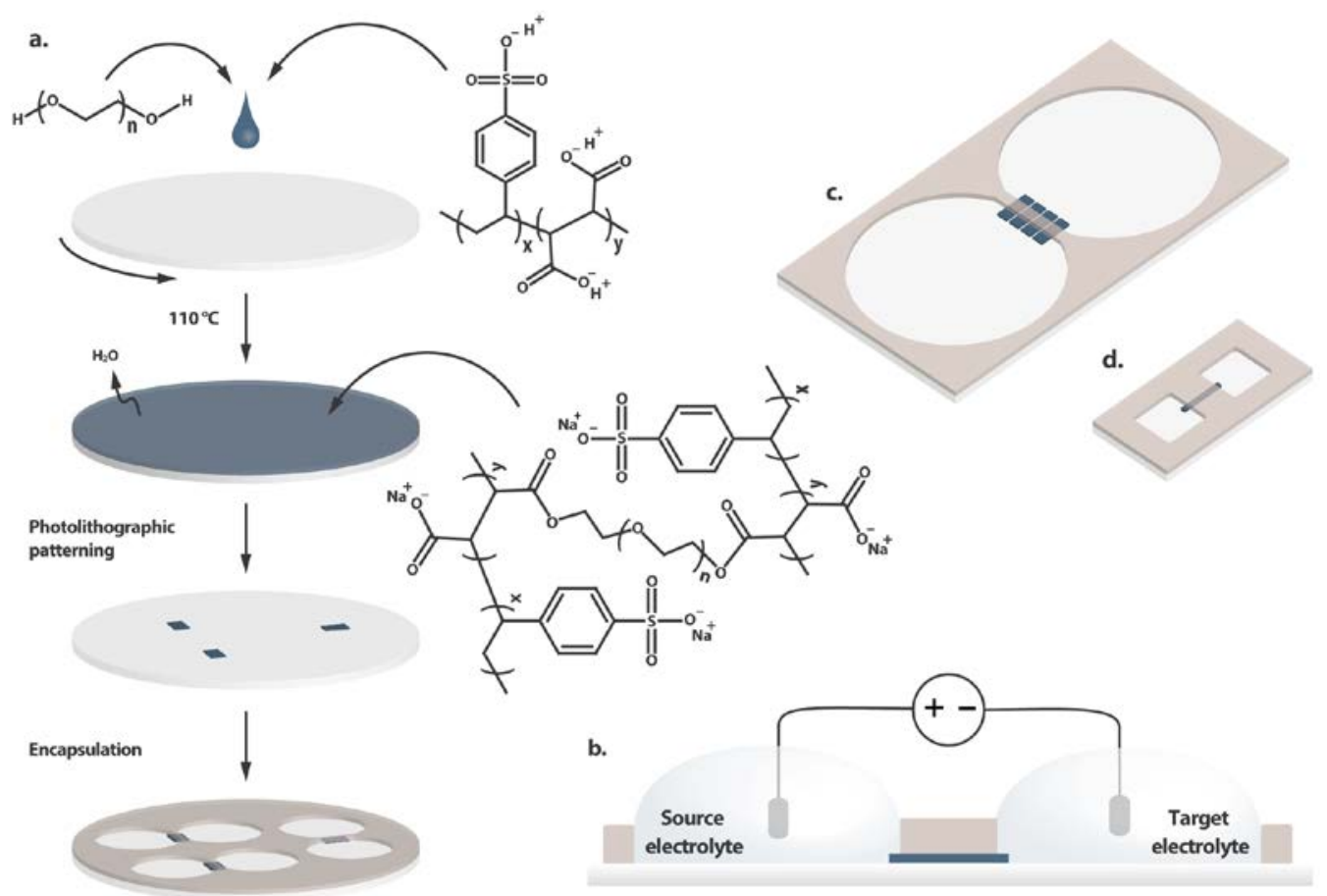

Figure 1. Fabrication and schematics of the organic electronic ion pump (OEIP). (a) PSS-co-MA is mixed with PEG before deposition and cross-linked as the maleic acid (MA) (from PSS-coMA) forms ester bonds together with -OH groups from PEG. After cross-linking, the PSS-coMA/PEG is stable for further processing. (b) OEIP schematic side view. Cations are driven electrophoretically from the source electrolyte, through the cation selective PSS-co-MA/PEG channel (dark blue), toward the target electrolyte due to an applied potential. (c) Top view of a low-resistance OEIP with four $1 \mathrm{~mm}$-wide channels, for membrane potential measurements. (d) Top view of OEIP with $3 \mathrm{~mm}$ x $450 \mu \mathrm{m}$ channel used for transport characterization. 


\section{a}

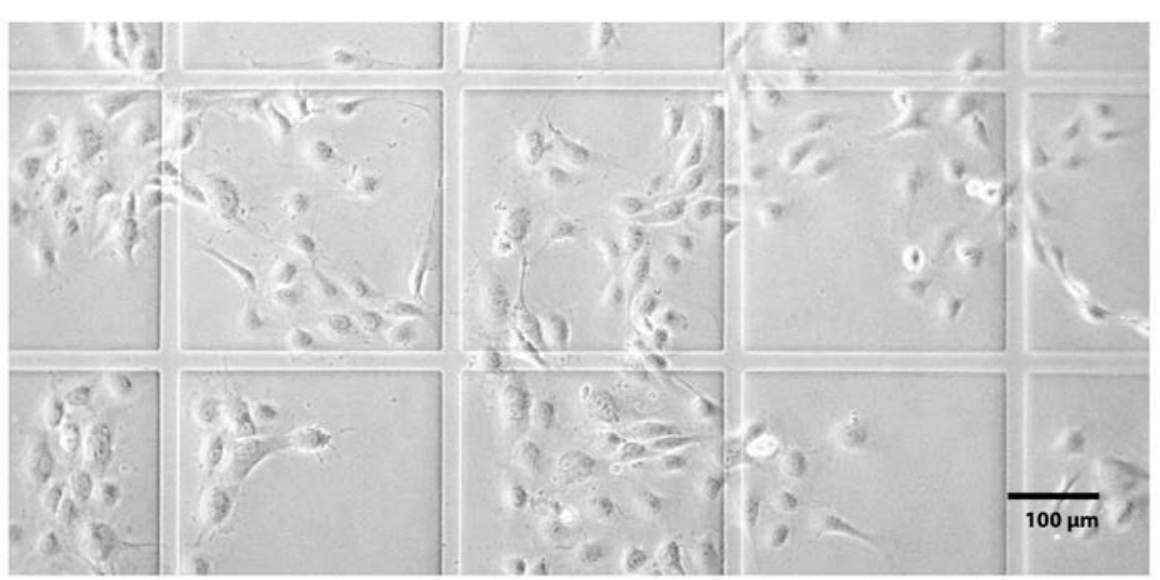

b

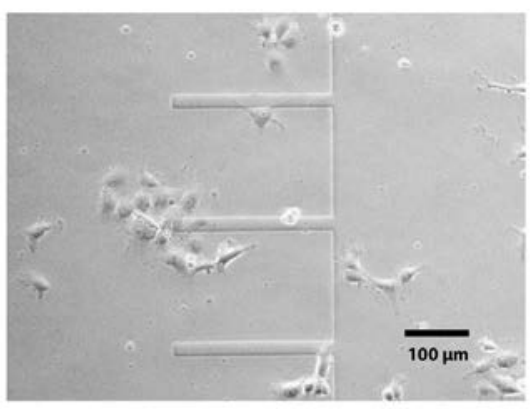

C

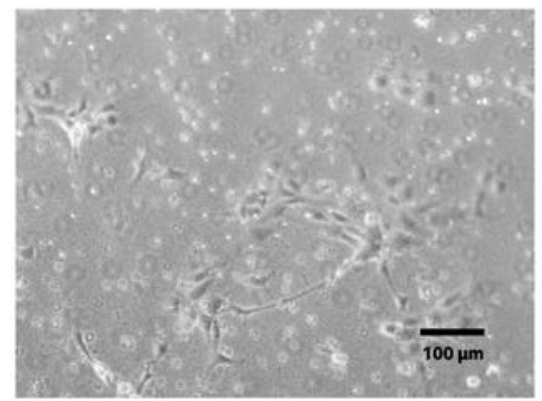

Figure 2. Human umbilical vein endothelial cells (HUVECs) seeded onto (a) test patterns of PSS-co-MA/PEG (300 $\mu \mathrm{m} \times 300 \mu \mathrm{m}$ squares) on glass; (b) multi-outlet OEIP of PSS-coMA/PEG on glass; and (c) dry-etched Orgacon ${ }^{\mathrm{TM}}$ foil (PET substrate). 


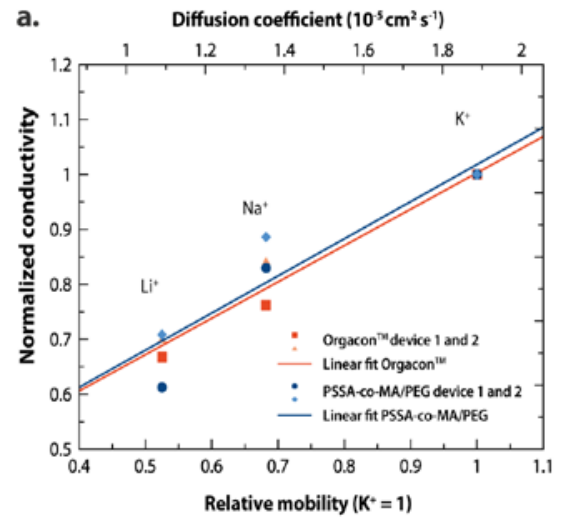

c.

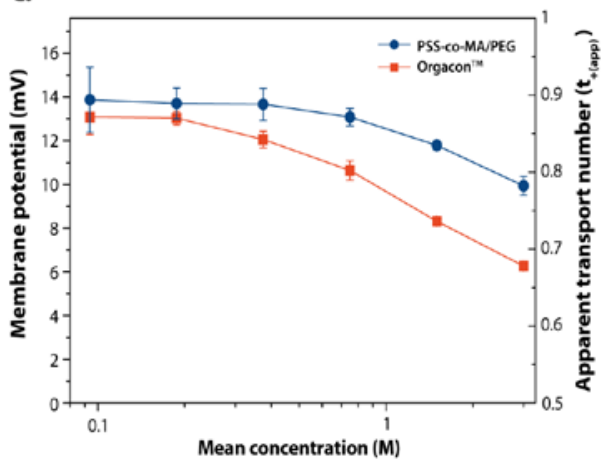

b. Diffusion coefficient $\left(10^{-5} \mathrm{~cm}^{2} \mathrm{~s}^{-1}\right)$

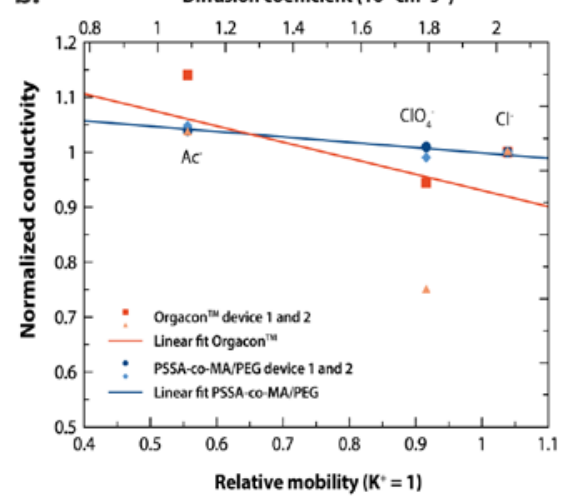

d.

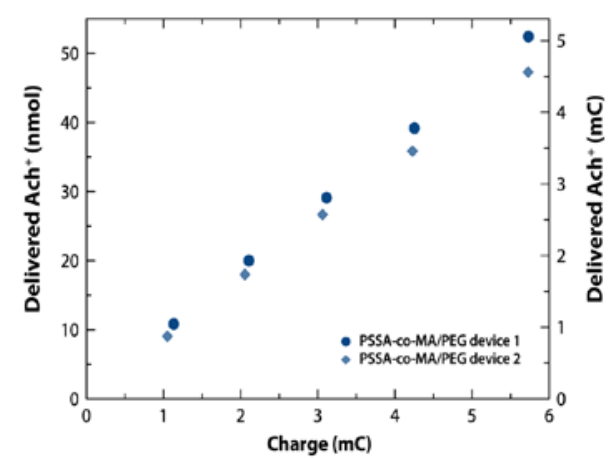

Figure 3. Transport characterization of PSS-co-MA/PEG. (a) Ionic conductivity vs. relative mobility $\left(\mathrm{K}^{+}=1\right)$ and diffusion coefficient in water for different source cations; $\mathrm{K}^{+}, \mathrm{Na}^{+}, \mathrm{Li}^{+}$with $\mathrm{Cl}^{-}$as target anion. (b) Ionic conductivity for different target anions; $\mathrm{Cl}^{-}, \mathrm{OAc}^{-}, \mathrm{ClO}_{4}{ }^{-}$with $\mathrm{K}+$ as source cation. In both a and b, results from two devices for each material are shown; blue symbols for Orgacon, red symbols for PSSA-co-MA/PEG. Each device response is normalized for comparison (see Supporting Information). (c) Membrane potential induced by concentration difference. One side of the channel had $2 \mathrm{x}$ concentration and the mean is given on the bottom axis. The mean and s.d. from six (PSSA-co-MA/PEG) and four (over-oxidized Orgacon $^{\mathrm{TM}}$ ) measurements are shown. (d) Direct characterization of acetylcholine transport through PSSAco-MA/PEG in two OEIPs (two shades of blue). 


\section{ASSOCIATED CONTENT}

\section{Supporting Information.}

Experimental details including device fabrication, cell culture and transport characterization (PDF).

\section{AUTHOR INFORMATION}

\section{Corresponding Author}

*e-mail: daniel.simon@liu.se

\section{Present Addresses}

† Department of Materials Science and Engineering, Stanford University 476 Lomita Mall, Stanford, CA 94305, USA

† Aix Marseille Université, Institut de Neurosciences des Systèmes, 13005 Marseille, France

\section{Notes}

K.T., M.B., and D.T.S. are shareholders in the small, researcher-controlled intellectual property company OBOE IPR AB (oboeipr.com), which owns patents related to this research.

\section{ACKNOWLEDGMENT}

The authors wish to thank Mats Sandberg for fruitful discussions and Jonas Christoffersson for cell characterization. Funding for this work was provided by the Knut and Alice Wallenberg Foundation (KAW Scholar, 2012.0302). Additional funding was provided by the Swedish 
Research Council (Vetenskapsrådet, 621-2011-3517), the Swedish Innovation Office (VINNOVA, 2010-00507), and the Önnesjö Foundation.

\section{ABBREVIATIONS}

IEM, ion exchange membrane; CEM, cation exchange membrane; AEM, anion exchange membrane; OEIP, organic electronic ion pump; IBMD, ion bipolar membrane diode; IBJT, ion bipolar junction transistor; PSS-co-MA, poly(4-styrenesulfonic acid-co-maleic acid); PEG, polyethylene glycol.

REFERENCES

(1) Durand, D. M.; Bikson, M. Suppression and Control of Epileptiform Activity by Electrical Stimulation: a Review. Proc. IEEE 2001, 89, 1065-1082.

(2) Benabid, A. L.; Chabardes, S.; Mitrofanis, J.; Pollak, P. Deep Brain Stimulation of the Subthalamic Nucleus for the Treatment of Parkinson's Disease. The Lancet Neurology 2009, 8, 67-81.

(3) Glajchen, M. Chronic Pain: Treatment Barriers and Strategies for Clinical Practice. J. Am. Board Fam. Pract. 2001, 14, 211-218.

(4) Ho, C. H.; Triolo, R. J.; Elias, A. L.; Kilgore, K. L.; DiMarco, A. F.; Bogie, K.; Vette, A. H.; Audu, M. L.; Kobetic, R.; Chang, S. R.; Chan, K. M.; Dukelow, S.; Bourbeau, D. J.; Brose, S. W.; Gustafson, K. J.; Kiss, Z. H. T.; Mushahwar, V. K. Functional Electrical Stimulation and Spinal Cord Injury. Phys Med Rehabil Clin N Am 2014, 25, 631-654. 
(5) Simon, D. T.; Kurup, S.; Larsson, K. C.; Hori, R.; Tybrandt, K.; Goiny, M.; Jager, E. W. H.; Berggren, M.; Canlon, B.; Richter-Dahlfors, A. Organic Electronics for Precise Delivery of Neurotransmitters to Modulate Mammalian Sensory Function. Nat. Mater. 2009, 8, 742-746.

(6) Jonsson, A.; Song, Z.; Nilsson, D.; Meyerson, B. A.; Simon, D. T.; Linderoth, B.; Berggren, M. Therapy Using Implanted Organic Bioelectronics. Sci. Adv. 2015, 1 (4), e1500039.

(7) Chun, H.; Chung, T. D. Iontronics. Annual Rev. Anal. Chem. 2015, 8, 441-462.

(8) Tybrandt, K.; Larsson, K. C.; Kurup, S.; Simon, D. T.; Kjäll, P.; Isaksson, J.; Sandberg, M.; Jager, E. W. H.; Richter-Dahlfors, A.; Berggren, M. Translating Electronic Currents to Precise Acetylcholine-Induced Neuronal Signaling Using an Organic Electrophoretic Delivery Device. Adv Mater 2009, 21 (44), 4442-4446.

(9) Williamson, A.; Rivnay, J.; Kergoat, L.; Jonsson, A.; Inal, S.; Uguz, I.; Ferro, M.; Ivanov, A.; Arbring Sjöström, T.; Simon, D. T.; Berggren, M.; Malliaras, G. G.; Bernard, C. Controlling Epileptiform Activity with Organic Electronic Ion Pumps. Adv. Mater. 2015, 27, $3138-3144$.

(10) Poxson, D. J.; Karady, M.; Gabrielsson, R.; Alkattan, A. Y.; Gustavsson, A.; Doyle, S. M.; Robert, S.; Ljung, K.; Grebe, M.; Simon, D. T.; Berggren, M. Regulating Plant Physiology with Organic Electronics. Proc. Natl. Acad. Sci. U.S.A. 2017, 116, 201617758.

(11) Han, J.-H.; Kim, K. B.; Bae, J. H.; Kim, B. J.; Kang, C. M.; Kim, H. C.; Chung, T. D. Ion Flow Crossing Over a Polyelectrolyte Diode on a Microfluidic Chip. Small 2011, 7, 2629-2639.

(12) Gabrielsson, E. O.; Berggren, M. Polyphosphonium-Based Bipolar Membranes for Rectification of Ionic Currents. Biomicrofluidics 2013, 7, 064117. 
(13) Tybrandt, K.; Larsson, K. C.; Richter-Dahlfors, A.; Berggren, M. Ion Bipolar Junction Transistors. Proc. Natl. Acad. Sci. U. S. A. 2010, 107, 9929-9932.

(14) Tybrandt, K.; Gabrielsson, E. O.; Berggren, M. Toward Complementary Ionic Circuits: the Npn Ion Bipolar Junction Transistor. J. Am. Chem. Soc. 2011, 133, 10141-10145.

(15) Gabrielsson, E. O.; Janson, P.; Tybrandt, K.; Simon, D. T.; Berggren, M. A Four-Diode Full-Wave Ionic Current Rectifier Based on Bipolar Membranes: Overcoming the Limit of Electrode Capacity. Adv. Mater. 2014, 26, 5143-5147.

(16) Tybrandt, K.; Forchheimer, R.; Berggren, M. Logic Gates Based on Ion Transistors. Nat. Commun. 2012, 3, 1-6.

(17) Jonsson, A.; Arbring Sjöström, T.; Tybrandt, K.; Berggren, M.; Simon, D. T. Chemical Delivery Array with Millisecond Neurotransmitter Release. Sci. Adv. 2016, 2, e1601340.

(18) Ye, Y.-S.; Rick, J.; Hwang, B.-J. Water Soluble Polymers as Proton Exchange Membranes for Fuel Cells. Polymers 2012, 4, 913-963.

(19) Nitanan, T.; Akkaramongkolporn, P.; Rojanarata, T.; Ngawhirunpat, T.; Opanasopit, P. Neomycin-Loaded Poly(Styrene Sulfonic Acid-Co-Maleic Acid) (PSSA-MA)/Polyvinyl Alcohol (PVA) Ion Exchange Nanofibers for Wound Dressing Materials. Int. J. Pharm. 2013, 448, 71-78.

(20) Devrim, Y. G.; Rzaev, Z.; Pişkin, E. Physically and Chemically Cross-Linked Poly\{[(Maleic Anhydride)-Alt-Styrene]-Co-(2-Acrylamido-2-Methyl-1-Propanesulfonic Acid)\}/Poly(Ethylene Glycol) Proton-Exchange Membranes. Macromol. Chem. Phys. 2007, 208 ,175-187. 
(21) Knop, K.; Hoogenboom, R.; Fischer, D.; Schubert, U. S. Poly(Ethylene Glycol) in Drug Delivery: Pros and Cons as Well as Potential Alternatives. Angew. Chem. Int. Ed. 2010, 49, $6288-6308$.

(22) Kontturi, K.; Murtomäki, L.; Manzanares, J. A. Ionic Transport Processes; Oxford University Press, 2008.

(23) Miyoshi, H. Diffusion Coefficients of Ions Through Ion-Exchange Membranes for Donnan Dialysis Using Ions of the Same Valence. Chem. Eng. Sci. 1997, 52, 1087-1096.

(24) Tanaka, Y. Ion Exchange Membranes; Elsevier, 2015.

(25) Kumar, M.; Khan, M. A.; Al-Othman, Z. A.; Choong, T. S. Y. Recent Developments in Ion-Exchange Membranes and Their Applications in Electrochemical Processes for in situIon Substitutions, Separation and Water Splitting. Sep. Purif. Rev. 2013, 42, 187-261.

(26) Vyas, P. V.; Ray, P.; Rangarajan, R.; Adhikary, S. K. Electrical Conductance of Heterogeneous Cation-Exchange Membranes in Electrolyte Solutions. J Phys Chem B 2002, 106, $11910-11915$.

(27) Vanýsek, P. Ionic Conductivity and Diffusion at Infinite Dilution. In CRC Handbook of Chemistry and Physics; Haynes, W. M., Ed.; CRC Press, 2014.

(28) Geise, G. M.; Cassady, H. J.; Paul, D. R.; Logan, B. E.; Hickner, M. A. Specific Ion Effects on Membrane Potential and the Permselectivity of Ion Exchange Membranes. Phys Chem Chem Phys 2014, 16, 21673-21681.

(29) Masaro, L.; Zhu, X. X. Physical Models of Diffusion for Polymer Solutions, Gels and Solids. Prog. Polym. Sci. 1998, 24, 731-775. 
(30) Lakshminarayanaiah, N. Counterion Transference Numbers in Ion-Exchange Membranes. J. Phys. Chem. 1969, 73, 97-102.

(31) Compan, V.; Sorensen, T. S.; Rivera, S. R. Comparison of Initial Time and Stationary State Measurements of the Emf of Concentration Cells Using Phenolsulfonic Acid Membrane Separators. J. Phys. Chem. 1995, 99, 12553-12558.

(32) Kumins, C. A.; London, A. The Electrochemistry of Vinyl Polymer Membranes. J. Polym. Sci. 1960, 46, 395-408.

(33) Kim, B.; Heo, J.; Kwon, H. J.; Cho, S. J.; Han, J.; Kim, S. J.; Lim, G. Tunable Ionic Transport for a Triangular Nanochannel in a Polymeric Nanofluidic System. ACS Nano 2013, 7, $740-747$.

(34) Chun, H.; Chung, T. D.; Ramsey, J. M. High Yield Sample Preconcentration Using a Highly Ion-Conductive Charge-Selective Polymer. Anal Chem 2010, 82, 6287-6292. 


\section{TABLE OF CONTENTS FIGURE}

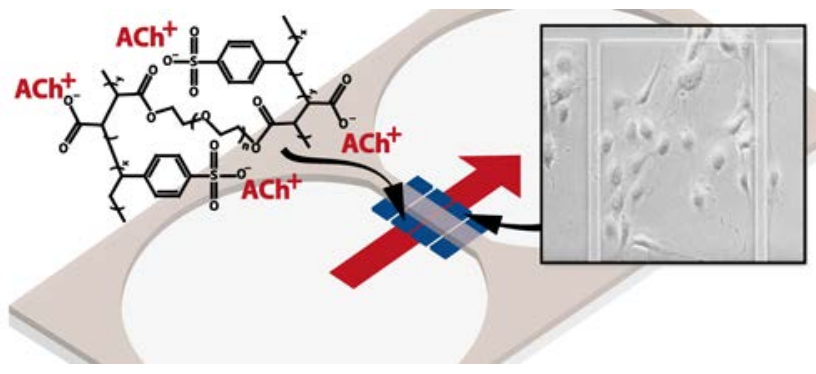

